\title{
PCB AND DIOXIN LEVELS IN PLASMA AND HUMAN MILK OF 418 DUTCH WOMEN AND THEIR INFANTS. PREDICTIVE VALUE OF PCB CONGENER LEVELS IN MATERNAL PLASMA FOR FETAL AND INFANT'S EXPOSURE TO PCBs AND DIOXINS.
}

\author{
C. Koopman-Esseboom ${ }^{1}$, M. Huisman ${ }^{2}$, N. Weisglas-Kuperus ${ }^{1}$, C.G. Van der Paauw ${ }^{3}$, L.G.M.Th. Tuinstra ${ }^{4}$, \\ E.R. Boersma², P.J.J. Sauer'.
}

1 Department of Paediatrics, Division of Neonatology, Erasmus University and University Hospital/Sophia Children's Hospital, Dr Molewaterplein 60,3015 GJ Rotterdam, the Netherlands.

2 Department of Obstetrics and Gynaecology, University of Groningen, the Netherlands.

3 TNO Nutrition and Food Research, Zeist, the Netherlands.

4 DLO State Institute for Quality Control of Agricultural Products, Wageningen, the Netherlands.

(Received in Sweden 1 February 1994; accepted 16 March 1994)

\begin{abstract}
Polychlorinated biphenyls (PCBs) as well as dioxins (polychlorinated dibenzo-p-dioxins (PCDDs) and dibenzofurans (PCDFs)) are potentially hazardous compounds in the environment for human beings.

In order to investigate PCB and dioxin exposure of Dutch women and their neonates, levels were examined in 418 mother-infant pairs. Four non-planar PCB congener levels (РCB 118, 138, 153 and 180) were measured in maternal plasma and in umbilical cord plasma. The 209 mothers who breast-fed their infants collected human milk samples for the analysis of seventeen 2,3,7,8-substituted PCDD and PCDF congener levels, three planar PCB and twenty-three non-planar PCB congener levels.

The dioxin and planar PCB levels we measured in human milk (mean 30 respectively $16 \mathrm{pg}$ TEQ/g fat), belong to the highest background levels analysed all over the world but they are in the normal range for highly industrialised, densely populated countries in Western Europe.

Correlation coefficients between PCB 118, 138, 153 and 180 congener levels in maternal plasma and PCB levels in cord plasma or PCB and dioxin levels in human milk are highly significant. However, the $95 \%$ predictive interval is too wide to predict accurately the PCB and dioxin levels to which an individual infant is exposed in utero or postnatally by breast-feeding, from the PCB levels in maternal plasma.
\end{abstract}

\section{Introduction}

Polychlorinated biphenyls (PCBs) are a compilation of 209 possible congeners with different chlorine substitutions. Within the group of PCB congeners different subgroups can be defined (Figure I). The planar PCBs have no chlorine atom on the ortho position of the biphenyl ring structure and they resemble closely the dioxins because of their planar structure. The mono-ortho PCBs have one chlorine atom on the ortho position and the di-ortho 


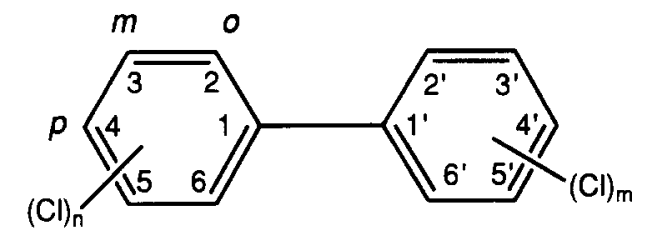

\section{Polychlorinated biphenyls (PCBs)}

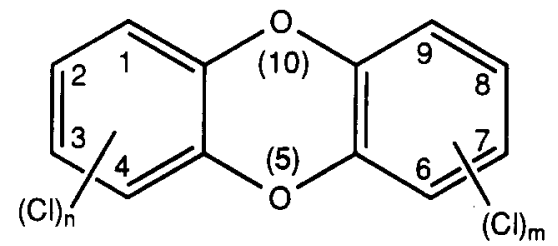

\section{Polychlorinated dibenzo-p-dioxins (PCDDs)}

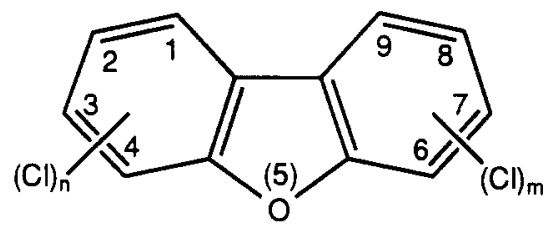

\section{Polychlorinated dibenzofurans (PCDFs)}

* $\mathrm{O}=$ Ortho, $\mathrm{M}=$ Meta, $\mathrm{P}=$ Para position.

PCBs have two chlorine atoms on the ortho position. All three kinds of PCB structures are also chlorinated in both para and at least two meta positions. Having more chlorine atoms on the ortho positions the coplanarity between the two phenyl rings is reduced and thus the toxicity of the congener.

The degree of biphenyl chlorination also alters the properties and applications of commercial mixtures. ${ }^{1}$ After the detection of ubiquitous levels of PCBs in the environment, the production and use of these compounds were banned in the late 1970s. However, large amounts of PCBs were dumped so that land, water and especially sludge can still be highly polluted and thus form a source of contamination for animals and humans.

Polychlorinated dibenzo-p-dioxins (PCDDs) and dibenzofurans (PCDFs), summarized as dioxins, are tricyclic aromatic compounds. Since the number of chlorine atoms can vary between 1 and 8 , there are potentially 75 different PCDD and 135 PCDF congeners. They are mainly formed as by-products during the combustion of municipal and hazardous waste and during the manufacturing of organochlorine chemicals. ${ }^{2}$

Higher chlorinated PCB and dioxin congeners are more abundant in most biotic samples because they are highly lipophilic, chemically stable and accumulate in the food chain. For humans the main sources of exposure to these compounds are dairy products, meat and fish. ${ }^{3}$ PCBs, PCDDs and PCDFs may produce a wide spectrum of toxic effects in animals and humans. ${ }^{4-10}$

In this paper we wish to answer the following questions: what are the PCB levels in maternal and cord plasma, 
and what are the PCB, PCDD and PCDF levels in human milk of Dutch women? Is there a decline in PCB or dioxin levels in human milk samples of the second and sixth week after delivery? As dioxin measurements are time-consuming, expensive and require large volumes of blood $(100-200 \mathrm{ml})$, we investigated if one of the more easily measurable PCB congener levels in maternal plasma could predict the PCB and dioxin exposure of the developing fetus and breast-fed infant.

This study is part of the Dutch PCB/Dioxin study, a prospective longitudinal study on possible adverse health effects of these pollutants for human beings.

\section{Methods}

The study group consisted of 418 healthy mother-infant pairs recruited between June 1990 and June 1992. Two hundred and seven pairs have been living in Rotterdam and the surrounding area, which is a highly industrialised region in the western part of the Netherlands. The other 211 pairs have been living in Groningen and the surrounding area, which is a semi-urban region in the north. Women were asked by their obstetrician or midwife to volunteer for the study. During the last month of pregnancy, mothers were visited at home for an explanation of the study protocol.

In order to establish an optimal study population, only infants born at term (37 to 42 weeks of gestation) without congenital anomalies or diseases were included. Pregnancy and delivery had to be passed without overt signs of serious illness or complications. All mothers and infants were of the Caucasian race. In order to study the effects of postnatal dioxin and PCB exposure a group of pregnant women was selected of whom $50 \%$ had the intention to breast-feed their infants and the other $50 \%$ to give formula feeding. Formula countains negligible levels of dioxins and PCBs. The mothers who breast-fed their infants $(n=209)$ had to do so for at least 6 weeks. The bottle-fed infants $(n=209)$ received formula milk from one batch during 7 months.

A blood sample was taken from the mothers in the last month of their pregnancy (36th to 40th week) for the measurement of PCB congener levels 2,3',4,4',5-pentachlorobiphenyl (PCB 118), 2, 2'3,4,4',5'-hexachlorobiphenyl (PCB 138), 2,2',4,4',5,5'-hexachlorobiphenyl (PCB 153) and 2,2',3,4,4',5,5'-heptachlorobiphenyl (PCB 180). ${ }^{11} \mathrm{~A}$ blood sample of the umbilical cord was taken to measure the same $\mathrm{PCB}$ congener levels as an indication of prenatal exposure. Blood was collected in a vacuum system EDTA-tube, transported in a cooling-pail, and centrifuged within 24 hours during 15 minutes at $3000 \mathrm{rpm}$; plasma was stored at $-20{ }^{\circ} \mathrm{C}$ until analysis.

In the second and sixth week after delivery, the mothers collected a 24-hour representative sample of breastmilk by collecting before each feeding as much milk as possible from both breasts with a vacuum pump. An aliquot of ten percent of each sample was pooled and stored in the refrigerator, the remainder was given to the infants in a bottle. After 24 hours the milk was stored at $-20^{\circ} \mathrm{C}$ until analysis.

PCB congeners in plasma were measured by gas chromatography with electron capture detection (GC-ECD). ${ }^{12}$ Plasma was denaturated with methanol and the chlorinated biphenyl congeners were isolated from the plasma with hexane-ethyl ether. The extract was dried over sodium sulphate, concentrated and purified by chromatography over basic alumina deactivated with $10 \%$ water. The analysis was carried out on two capillary columns of different polarity. The limit of determination for the four congeners was $0.01 \mathrm{ng} / \mathrm{g}$ plasma. The recovery of the 
chlorinated biphenyl congeners added to the plasma before extraction and determined as described above, was $>95 \%$. In each series a chemical blank and a control sample have been analysed to assure the quality.

The human milk samples were analysed for the 17 most abundant 2,3,7,8-substituted PCDD and PCDF congeners and three planar PCB congeners (3, 3', 4, $4^{\prime}$-tetrachlorobiphenyl (PCB 77), 3, 3',4,4',5-pentachlorobiphenyl (PCB 126), and 3,3',4,4',5,5'-hexachlorobiphenyl(PCB 169)) by gas chromatography-high-resolution mass spectrometry (GC-HRMS). ${ }^{13}$ Twenty-three non-planar PCB congeners (IUPAC no. 28, 52, 66, 70, 99, 101, 105, 118, 128, $137,138,141,151,153,156,170,177,180,183,187,194,195$ and 202) ${ }^{11}$ were measured by GC-ECD. ${ }^{14}$

To express the toxic potency of the mixture of dioxins and PCBs in breastmilk samples, the international toxic equivalence factor (I-TEF) approach ${ }^{15}$ was used for the PCDDs and PCDFs, and the WHO 1993 approach for the PCBs. ${ }^{16} \mathrm{~A}$ TEF value was assigned to the dioxin and dioxin-like PCB congeners which represents their relative toxic potency towards 2,3,7,8-TCDD, the most toxic dioxin congener which TEF value is one. By multiplying the concentration (pg/g milkfat) and TEF value, the toxic equivalent (TEQ) of each congener was calculated (pg TEQ/g milkfat). By adding up the TEQs of all congeners the total-TEQ value was obtained. The TEQ-sum of the 17 dioxin congeners yielded a dioxin-TEQ, the three planar PCB $(77,126,169)$, three mono-ortho PCB (105, $118,156)$ and two di-ortho PCB congeners $(170,180)$ revealed a planar, mono-ortho and di-ortho PCB-TEQ respectively. The other $\mathrm{PCB}$ congeners did not receive a TEF value because they have negligible dioxin-like activity. The study protocol had been approved by the medical ethical committee of the University Hospital Rotterdam/Sophia Children's Hospital and the University of Groningen. Informed consent had been given by the parents.

Data analysis was performed by means of the statistical soft-ware package SPSS/PC. Means, medians, ranges and standard deviations are reported in terms of original distributions. Spearman rank correlation coefficients were measured between the different congener levels in plasma and human milk. A 95\% interval for predicting the total-TEQ in human milk from the PCB 153 level in maternal plasma for an individual subject, was calculated after $\log$ transformation of the variables. The paired Student's $t$ test was used to determine differences in mean PCB and dioxin levels in paired human milk samples of the second and sixth week, after log transformation.

\section{Results}

The mean, median and range of PCB 118, 138, 153 and 180 congener levels in maternal and cord plasma are summarized in Table I. Three maternal plasma samples missed, 36 cord plasma samples missed for the PCB 138, 153 and 180 analysis and 45 for the PCB 118 analysis, due to organization failure.

The mean, median, range and available TEQ values of all PCDD, PCDF and PCB congener levels in human milk samples of the second week after birth are summarized in Table II and III. Representative PCDD, PCDF and planar PCB congener levels are presented of 176 respectively 194 human milk samples and non-planar PCB congener levels of 195 samples.

Spearman rank correlation coefficients between levels of the four different non-planar PCB congeners $(118,138$, 153, 180) within one biological sample such as maternal plasma, cord plasma or human milk are all highly significant and vary from 0.71 to 0.98 . However, the correlation coefficients in human milk between all other 
PCB and dioxin congener levels vary considerably from -0.16 to 0.85 .

Table I Mean, median and range of PCB 118, 138, 153 and 180 levels (ng/g plasma) in maternal and cord plasma.

\begin{tabular}{lcccc}
\hline & PCB 118 & PCB 138 & PCB 153 & PCB 180 \\
\cline { 2 - 5 } Maternal plasma & 415 & 415 & 415 & 415 \\
$\mathrm{n}$ & 0.16 & 0.60 & 0.91 & 0.54 \\
mean & 0.15 & 0.56 & 0.84 & 0.50 \\
median & $0.02-0.60$ & $0.13-1.60$ & $0.18-2.50$ & $0.08-3.10$ \\
range & & & & \\
\hline Cord plasma & 373 & 382 & 382 & 382 \\
$\mathrm{n}$ & 0.04 & 0.13 & 0.18 & 0.10 \\
mean & 0.04 & 0.11 & 0.15 & 0.08 \\
median & $0.01-0.16$ & $0.01-0.59$ & $0.02-0.85$ & $0.01-0.76$ \\
range & & & & \\
\hline
\end{tabular}

Relations between PCB and dioxin congener levels in different biological samples were also analysed. The correlation coefficients between levels of the four PCB congeners 118, 138, 153 and 180 in maternal plasma and cord plasma vary from 0.52 to 0.74 . In Table IV Spearman rank correlation coefficients between these four PCB congener levels in maternal plasma and human milk are presented. The correlation coefficients are all significant, the corresponding congeners having the highest correlations. In Table V Spearman rank correlation coefficients between these four PCB congener levels in human milk and dioxin- and PCB-TEQ levels in human milk have been summarized. The correlation coefficients are highly significant. In Table VI Spearman rank correlation coefficients between these PCB congener levels in maternal plasma and dioxin-TEQ and PCB-TEQ levels in human milk are summarized. The correlation coefficients are less compared to the coefficients in Table V. In Figure II the $95 \%$ confidence interval for the regression line between the PCB 153 level in maternal plasma and the total-TEQ level in human milk is shown as well as the $95 \%$ predictive interval for the total-TEQ from the PCB 153 level for an individual subject. For instance, if the PCB 153 level in maternal plasma would be equal to the mean, $0.91 \mathrm{ng} / \mathrm{g}$ plasma, the upper limit of the $95 \%$ predictive interval of the total-TEQ level in her breastmilk would be 100.0 $\mathrm{pg} \mathrm{TEQ} / \mathrm{g}$ fat, the lower limit $39.8 \mathrm{pg}$ TEQ/g fat. Since the predictive interval is wide, it is impossible in this way to predict accurately the PCB and dioxin levels to which an individual fetus or breast-fed infant is exposed.

Paired human milk samples collected in the second and sixth week after birth were analyzed for dioxin- and PCBTEQ levels. Results are presented in Table VII. The Student's $t$ test for paired samples showed no significant decrease in mean dioxin-, planar PCB- or total-TEQ level over a four weeks period. However the mean monoortho and di-ortho PCB-TEQ level were significantly decreased in this period. 
Table II Mean, median and range (pg/g fat) and mean TEQ (pg TEQ/g fat) of PCDD, PCDF (N = 176) and planar PCB $(\mathrm{N}=194)$ levels in human milk samples of the second week after birth.

\begin{tabular}{|c|c|c|c|c|c|c|}
\hline PCDD'S & IUPAC & TEF & mean & median & range & TEQ \\
\hline $2,3,7,8-\mathrm{TCDD}$ & 48 & 1 & 4.0 & 3.6 & $0.03-15.8$ & 4.0 \\
\hline 1,2,3,7,8-PECDD & 54 & 0.5 & 10.6 & 10.2 & $0.1-30.1$ & 5.3 \\
\hline $1,2,3,4,7,8-\mathrm{HXCDD}$ & 66 & 0.1 & 8.7 & 8.7 & $0.04-28.4$ & 0.9 \\
\hline $1,2,3,6,7,8-\mathrm{HXCDD}$ & 67 & 0.1 & 47.4 & 45.8 & $18.7-131.8$ & 4.7 \\
\hline $1,2,3,7,8,9-\mathrm{HXCDD}$ & 70 & 0.1 & 6.7 & 6.7 & $0.04-21.0$ & 0.7 \\
\hline $1,2,3,4,6,7,8-\mathrm{HPCDD}$ & 73 & 0.01 & 63.2 & 58.1 & $0.1-178.4$ & 0.6 \\
\hline $1,2,3,4,6,7,8,9-\mathrm{OCDD}$ & 75 & 0.001 & 799.6 & 670.4 & $224.2-3113.6$ & 0.8 \\
\hline PCDF'S & IUPAC & TEF & mean & median & range & TEQ \\
\hline $2,3,7,8-\mathrm{TCDF}$ & 83 & 0.1 & 0.8 & 0.7 & $0.02-3.2$ & 0.08 \\
\hline $1,2,3,7,8-\mathrm{PECDF}$ & 94 & 0.05 & 0.3 & 0.1 & $0.02-4.7$ & 0.01 \\
\hline $2,3,4,7,8-\mathrm{PECDF}$ & 114 & 0.5 & 22.7 & 21.6 & $8.4-55.0$ & 11.3 \\
\hline $1,2,3,4,7,8-\mathrm{HXCDF}$ & 118 & 0.1 & 6.6 & 6.4 & $0.1-15.3$ & 0.7 \\
\hline $1,2,3,6,7,8-\mathrm{HXCDF}$ & 121 & 0.1 & 5.7 & 5.5 & $0.1-12.5$ & 0.6 \\
\hline $2,3,4,6,7,8-\mathrm{HXCDF}$ & 130 & 0.1 & 3.6 & 3.0 & $0.04-16.9$ & 0.4 \\
\hline $1,2,3,7,8,9-\mathrm{HXCDF}$ & 124 & 0.1 & 0.3 & 0.1 & $0.02-21.5$ & 0.03 \\
\hline $1,2,3,4,6,7,8-\mathrm{HPCDF}$ & 131 & 0.01 & 7.9 & 6.3 & $0.1-72.3$ & 0.08 \\
\hline $1,2,3,4,7,8,9-\mathrm{HPCDF}$ & 134 & 0.01 & 0.2 & 0.2 & $0.05-1.8$ & 0.0 \\
\hline $1,2,3,4,6,7,8,9-\mathrm{OCDF}$ & 135 & 0.001 & 2.2 & 0.4 & $0.1-215.5$ & 0.0 \\
\hline DIOXIN-TEQ & & & & & & 30.2 \\
\hline PLANAR PCB'S & IUPAC & TEF & mean & median & range & TEQ \\
\hline $3,3^{\prime}, 4,4^{\prime}-\mathrm{PCB}$ & 77 & 0.0005 & 19.3 & 14.8 & $3.7-143.2$ & 0.01 \\
\hline $3,3^{\prime}, 4,4^{\prime}, 5-\mathrm{PCB}$ & 126 & 0.1 & 152.0 & 137.5 & $39.4-443.9$ & 15.2 \\
\hline $3,3^{\prime}, 4^{\prime} 4^{\prime}, 5,5^{\prime}-\mathrm{PCB}$ & 169 & 0.01 & 84.3 & 79.5 & $33.2-282.9$ & 0.8 \\
\hline PLANAR PCB-TEQ & & & & & & 16.0 \\
\hline
\end{tabular}

IUPAC = International Union of Pure and Applied Chemistry

TEF = Toxic Equivalence Factor (I-TEFs for dioxins ${ }^{15}$, WHO '93 TEFs for PCBs ${ }^{16}$ )

TEQ $=$ Toxic Equivalent 

Mean, median and range (ng/g fat) and mean TEQ (pg TEQ/g fat) of non-planar PCB levels in human milk samples of the second week after birth. $N=195$.

\begin{tabular}{|c|c|c|c|c|c|c|}
\hline & IUPAC & TEF & mean & median & range & TEQ \\
\hline $2,4,4^{\prime}$ & 28 & & 12.1 & 5.8 & $0.2-188.6$ & \\
\hline $2,2^{\prime}, 5,5^{\prime}$ & 52 & & 2.6 & 1.5 & $0.2-32.7$ & \\
\hline $2,3^{\prime}, 4,4^{\prime}$ & 66 & & 11.6 & 9.4 & $0.4-79.6$ & \\
\hline $2,3,4^{\prime}, 5$ & 70 & & 18.5 & 17.4 & $0.5-54.2$ & \\
\hline $2,2^{\prime}, 4,4^{\prime}, 5$ & 99 & & 19.7 & 18.1 & $1.4-54.1$ & \\
\hline $2,2^{\prime}, 4,5,5^{\prime}$ & 101 & & 1.5 & 1.1 & $0.2-10.0$ & \\
\hline $2,3^{\prime} 3^{\prime}, 4,4^{\prime}$ & $105^{*}$ & 0.0001 & 9.4 & 8.7 & $0.4-22.9$ & 0.9 \\
\hline $2,3^{\prime}, 4,4^{\prime}, 5$ & $118^{*}$ & 0.0001 & 35.5 & 32.7 & $9.7-94.0$ & 3.6 \\
\hline $2,2^{\prime}, 3,3^{\prime}, 4,4^{\prime}$ & 128 & & 4.0 & 3.7 & $0.4-16.1$ & \\
\hline $2,2^{\prime}, 3,4,4^{\prime}, 5$ & 137 & & 16.8 & 13.4 & $1.4-174.6$ & \\
\hline $2,2^{\prime}, 3,4^{\prime}, 4^{\prime}, 5^{\prime}$ & 138 & & 129.9 & 124.2 & $43.8-314.3$ & \\
\hline $2,3,4,5,2^{\prime} 5^{\prime}$ & 141 & & 1.1 & 0.8 & $0.3-7.1$ & \\
\hline $2,2^{\prime}, 3,5,5^{\prime}, 6$ & 151 & & 0.9 & 0.8 & $0.2-7.5$ & \\
\hline $2,2^{\prime}, 4,4^{\prime}, 5,5^{\prime}$ & 153 & & 186.3 & 174.7 & $59.9-475.7$ & \\
\hline $2,3,3^{\prime}, 4^{\prime}, 4^{\prime}, 5$ & $156^{*}$ & 0.0005 & 21.0 & 20.1 & $2.9-78.0$ & 10.5 \\
\hline $2,2^{\prime}, 3,3^{\prime}, 4,4^{\prime}, 5$ & $170^{* *}$ & 0.0001 & 37.1 & 35.4 & $11.4-219.9$ & 3.7 \\
\hline $2,2^{\prime}, 3,3^{\prime}, 4^{\prime}, 5,6$ & 177 & & 6.3 & 5.9 & $0.4-22.4$ & \\
\hline $2,2^{\prime}, 3,4,4^{\prime}, 5,5^{\prime}$ & $180 * *$ & 0.00001 & 76.8 & 71.3 & $2.5-418.8$ & 0.8 \\
\hline $2,2^{\prime}, 3,4,4^{\prime}, 5^{\prime}, 6$ & 183 & & 12.2 & 11.5 & $2.9-34.6$ & \\
\hline $2,2^{\prime}, 3,4^{\prime}, 5,5^{\prime}, 6$ & 187 & & 20.0 & 17.9 & $2.9-103.8$ & \\
\hline $2,2^{\prime}, 3,3^{\prime}, 4,4^{\prime}, 5,5^{\prime}$ & 194 & & 8.6 & 7.8 & $0.6-74.2$ & \\
\hline $2,2^{\prime}, 3,3^{\prime}, 4^{\prime}, 4^{\prime}, 5,6$ & 195 & & 2.9 & 2.7 & $0.5-15.5$ & \\
\hline $2,2^{\prime}, 3,3^{\prime}, 5,5^{\prime}, 6,6^{\prime}$ & 202 & & 0.9 & 0.7 & $0.2-8.6$ & \\
\hline Mono-ortho PCB-TEQ & & & & & & 15.0 \\
\hline Di-ortho PCB-TEQ & & & & & & 4.5 \\
\hline
\end{tabular}

IUPAC $=$ International Union of Pure and Applied Chemistry TEF $=$ Toxic Equivalence Factor (WHO '93 TEFs for PCBs ${ }^{16}$ ) $\mathrm{TEQ}=$ Toxic Equivalent

* = mono-ortho PCB

** = di-ortho PCB 
Table IV Spearman rank correlation coefficients between PCB congener levels in human milk (ng/g fat) and PCB congener levels in maternal plasma (ng/g). All correlations have a significance of $\mathrm{p} \leq \mathbf{0 . 0 0 0 1}$. $N=193$.

\begin{tabular}{lcccc}
\hline & \multicolumn{4}{c}{ Maternal plasma } \\
\cline { 2 - 5 } Human milk & PCB 118 & PCB 138 & PCB 153 & PCB 180 \\
\cline { 2 - 5 } PCB 118 & 0.77 & 0.69 & 0.64 & 0.51 \\
PCB 138 & 0.63 & 0.79 & 0.76 & 0.67 \\
PCB 153 & 0.62 & 0.78 & 0.77 & 0.72 \\
PCB 180 & 0.48 & 0.63 & 0.66 & 0.70 \\
\hline
\end{tabular}

Table V Spearman rank correlation coefficients between PCB congener levels in human milk (ng/g fat) and dioxin and PCB levels in human milk (pg TEQ/g fat). All correlations have a significance of $p \leq 0.0001$.

\begin{tabular}{lccccc}
\hline & \multicolumn{5}{c}{ Human milk } \\
\cline { 2 - 6 } Human milk & dioxins & planar PCBs & $\begin{array}{c}\text { mono-ortho } \\
\text { PCBs }\end{array}$ & $\begin{array}{c}\text { di-ortho } \\
\text { PCBs }\end{array}$ & Total TEQ \\
PCB 118 & $\mathrm{n}=168$ & $\mathrm{n}=186$ & $\mathrm{n}=195$ & $\mathrm{n}=195$ & $\mathrm{n}=168$ \\
\cline { 2 - 6 } PCB 138 & 0.69 & 0.81 & 0.85 & 0.64 & 0.84 \\
PCB 153 & 0.77 & 0.61 & 0.93 & 0.86 & 0.85 \\
PCB 180 & 0.78 & 0.59 & 0.94 & 0.90 & 0.86 \\
\hline
\end{tabular}

(I-TEFs for dioxins ${ }^{15}$, WHO '93 TEFs for PCBs ${ }^{16}$ )

Table VI Spearman rank correlation coefficients between PCB congener levels in maternal plasma (ng/g) and dioxin and PCB levels in human milk (pg TEQ/g fat). All correlations have a significance of $p \leq 0.0001$.

\begin{tabular}{lccccc}
\hline & \multicolumn{5}{c}{ Human milk } \\
\cline { 2 - 6 } Maternal plasma & $\begin{array}{c}\text { dioxins } \\
\mathrm{n}=174\end{array}$ & $\begin{array}{c}\text { planar PCBs } \\
\mathrm{n}=192\end{array}$ & $\begin{array}{c}\text { mono-ortho PCBs } \\
\mathrm{n}=193\end{array}$ & $\begin{array}{c}\text { di-ortho PCBs } \\
\mathrm{n}=193\end{array}$ & $\begin{array}{c}\text { Total TEQ } \\
\mathrm{n}=166\end{array}$ \\
\cline { 2 - 6 } PCB 118 & 0.64 & 0.70 & 0.64 & 0.51 & 0.71 \\
PCB 138 & 0.65 & 0.51 & 0.75 & 0.69 & 0.71 \\
PCB 153 & 0.67 & 0.50 & 0.75 & 0.72 & 0.71 \\
PCB 180 & 0.63 & 0.42 & 0.70 & 0.76 & 0.66 \\
\hline
\end{tabular}

(I-TEFs for dioxins ${ }^{15}$, WHO '93 TEFs for PCBs ${ }^{16}$ ) 
Figure II $95 \%$ Predictive interval of the total-TEQ level in human milk from the PCB 153 level in maternal plasma.

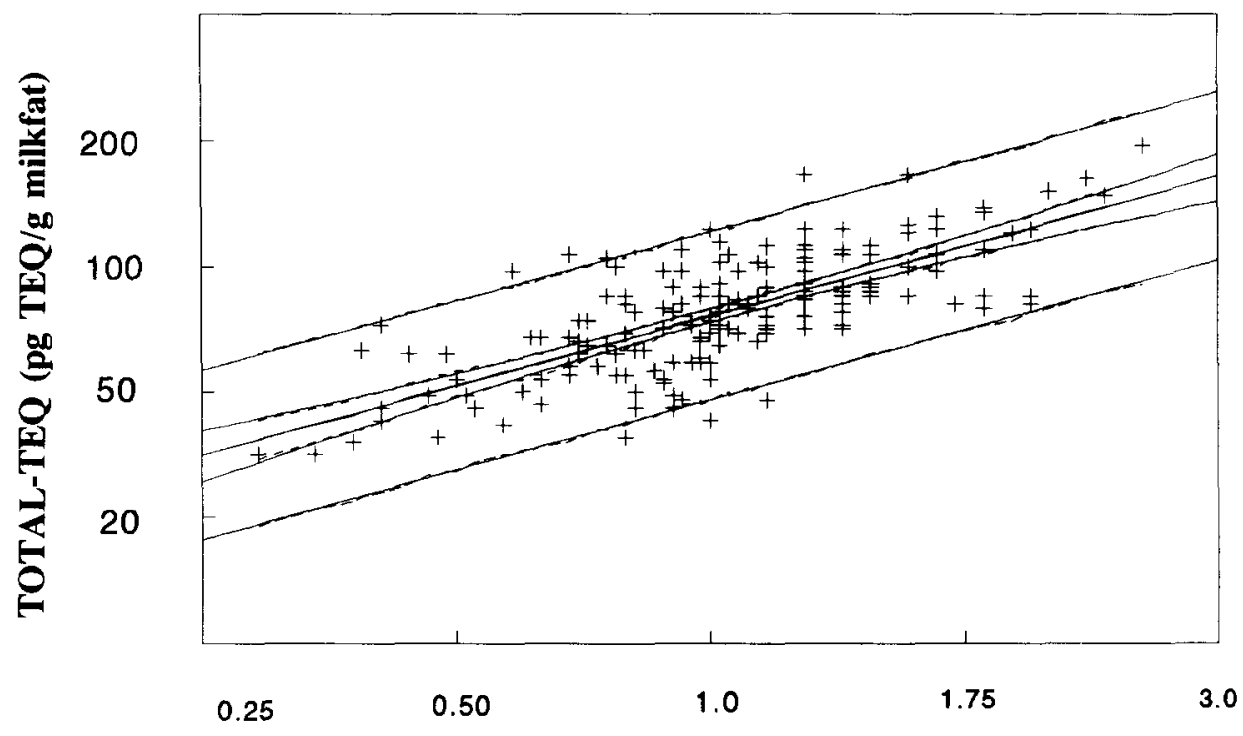

PCB 153 (ng/g plasma)

${ }^{*} \log Y=1.82+0.62 \log X, \quad r=0.73$

Table VII Mean dioxin, planar PCB, mono-ortho PCB, di-ortho PCB and total TEQ levels (pg TEQ/g fat) in human milk samples of the second and sixth week after delivery.

\begin{tabular}{|c|c|c|c|c|c|}
\hline & Sample I & Sample II & $\mathrm{P}^{*}$ & corr. coeff. ${ }^{* *}$ & $\mathbf{N}$ \\
\hline & mean s.d. & mean s.d. & & & \\
\hline dioxins & $34.4 \pm 12.4$ & $31.8 \pm 10.3$ & 0.07 & 0.88 & 27 \\
\hline planar PCBs & $16.7 \pm 8.0$ & $16.7 \pm 7.7$ & 0.91 & 0.67 & 44 \\
\hline mono-ortho PCBs & $15.2 \pm 5.8$ & $14.3 \pm 5.4$ & 0.002 & 0.85 & 180 \\
\hline di-ortho PCBs & $4.5 \pm 2.4$ & $4.3 \pm 2.3$ & 0.001 & 0.92 & 180 \\
\hline total TEQ & $72.3 \pm 31.3$ & $66.0 \pm 23.9$ & 0.10 & 0.94 & 19 \\
\hline
\end{tabular}

$*$ Paired Student's $t$ test, for log normalized values

** = Correlation coefficient between the paired milk samples of one woman

s.d. $=$ standard deviation 


\section{Discussion}

We measured 17 dioxin and 26 PCB congener levels in human milk and 4 PCB congener levels in maternal and cord plasma. The mean dioxin-TEQ level (30 pg TEQ/g fat) is elevated compared to dioxin background levels in the Scandinavian countries (20 pg TEQ/g fat), Spain (13 pg TEQ/g fat) and the United States (17 pg TEQ/g fat). However the dioxin and PCB levels found in this study are comparable to the levels in other highly industrialised, densely populated countries in Western Europe such as Belgium, The United Kingdom and the Federal Republic of Germany. ${ }^{17}$ In the Netherlands the National Institute of Public Health and Environmental Protection (RIVM), measured PCDD, PCDF and PCB congener levels in human milk, in a WHO coordinated international study. ${ }^{18}$ In 1983 only PCB congener levels (IUPAC no. 28, 52, 101, 118, 153, 138, 180 and 194) were measured. In 1988 both PCBs and dioxins were measured. The PCB levels tended to decrease over the 5 years period, as is the tendency all over the world. The median PCB congener levels we measured from 1990 to 1992 are somewhat higher (PCB 138 and 153) or similar (PCB 118, 180 and 194) to the results the RIVM presented over the year 1983 . This may be due to differences in analytical methods.

PCB congener levels in maternal plasma are about five times higher compared to the levels in umbilical cord plasma. When expressed on a lipid base the levels are comparable ${ }^{19}$, because PCBs are highly bound to lipids which contents in cord blood are low compared to maternal blood..$^{20,21}$

The total-TEQ value and the contribution of individual dioxin and PCB congener levels to the TEQ value, depend on the TEF values used. According to the model we used, the dioxins contribute for $46 \%$, the planar PCBs for $24 \%$, the mono-ortho PCBs for $23 \%$ and the di-ortho PCBs for $7 \%$ to the total-TEQ value (65.2 pg TEQ/g fat). Although the mono-ortho and di-ortho PCBs have low TEFs, they can contribute considerably to the total-TEQ because of their high concentrations in human milk.

Correlation coefficients between the non-planar PCB congener levels (PCB 118, 138, 153 and 180) in maternal plasma, cord plasma and human milk are quite high within one biological sample as plasma or human milk $(0.71$ to 0.98), as well as between different biological samples. However, correlation coefficients between other PCB and dioxin congener levels in human milk differ considerably. One explanation for this wide range in correlation coefficients could be that different congeners have different half-lives. Secondly, due to limitations of determination, levels of some congeners in plasma as well as in human milk might be less accurate. Finally, correlation coefficients between non-planar PCB levels in maternal plasma and dioxin- or PCB-TEQ levels in human milk are lower compared to the correlation coefficients between the same non-planar PCB congener levels in human milk and the TEQ levels in human milk. Levels in plasma are detected in ng/g plasma while levels in human milk are expressed as ng/g fat. However, when we correlated the PCB plasma levels with the TEQ levels in human milk on product base (ng/g milk), the correlation coefficients were lower. Since new fat is produced in the breast, plasma and milk might be different pools for PCB and dioxin congeners.

We found a decline in mono-ortho and di-ortho PCB-TEQ levels in human milk over a 4 weeks period. However, the dioxin-, planar PCB- and total-TEQ levels did not change in this period. Fürst ${ }^{22}$ and Norén ${ }^{23}$ did report a decline in dioxin levels over a longer period of breast-feeding.

Non-planar PCB levels in maternal plasma are relatively easy and cheap to analyse instead of dioxin and PCB 
levels in human milk, which are time-consuming, expensive measurements. Moreover it is easier to collect a blood sample than a 24-hour representative human milk sample which is an onerous task for the mothers who collect the milk. Our results demonstrate that non-planar PCB analysis in maternal plasma during pregnancy or even before conception can give an indication about in utero and postnatal breast-feeding exposure to PCDDs, PCDFs and PCBs, but the $95 \%$ predictive interval for an individual infant is wide.

\section{Acknowledgements}

This study was supported by the Dutch Toxicology Research Promotion Programme and the Health Research Stimulation Programme. Wim Hop is kindly acknowledged for statistical assistance, Joost de Jongh for preparing Figure I and Jan Raatgever for drawing Figure II.

\section{References}

1 De Voogt P, Brinkman UATh. Production, properties and usage of polychlorinated biphenyls. In: Kimbrough RD, Jensen AA (eds). Halogenated biphenyls, terphenyls, naphthalenes, dibenzodioxins and related products. Elsevier 1989, pp 3-45.

2 Rappe C, Andersson R, Bergquist PA et al. Overview of environmental fate of chlorinated dioxins and dibenzofurans, sources, levels and isomeric pattern in various matrices. Chemosphere 1987; 16: 1603-1618.

3 Theelen RMC. Modeling of human exposure to TCDD and I-TEQ in the Netherlands: Background and occupational. In: Gallo MA, Scheuplein RJ, Van der Heijden KA (eds). Biological basis for risk assessment of dioxins and related compounds. Cold Spring Harbor Laboratory Press; Banbury report 1991; 35: $277-290$.

Safe S. Polychlorinated biphenyls (PCBs) and polybrominated biphenyls (PBBs): biochemistry, toxicology, and mechanism of action. CRC Crit Rev Toxicol 1984; 13 (4): 319-395.

5 Skene SA, Dewhurst IC, Greenberg M. Polychlorinated dibenzo-p-dioxins and polychlorinated dibenzofurans: The risks to human health. A review. Human Toxicol 1989; 8: 173-203.

6 Murai K, Okamura K, Tsuji H, Kajiwara E, Watanabe H, Akagi K, Fujishina. Thyroid function in "Yusho" patients exposed to polychlorinated biphenyls. Environ Res 1987; 44: 179-187.

7 Koopman-Esseboom C, Morse DC, Weisglas-Kuperus N, Lutke-Schipholt I, Van der Paauw CG, Tuinstra LGMTh, Brouwer A, Sauer PJJ. Effects of dioxins and polychlorinated biphenyls on thyroid hormone status of pregnant women and their infants. (Submitted)

8 Rogan WJ, Gladen BC, Hung K-L, Koong S-L, Shih L-Y, Taylor JS, Wu Y-C, Yang D, Ragan NB, Hsu C-C. Congenital poisoning by polychlorinated biphenyls and their contaminants in Taiwan. Science 1988; 241: 334-336.

9 Jacobson SW, Fein GG, Jacobson JL, Schwartz PM, Dowler JK. The effect of intrauterine PCB exposure on visual recognition memory. Child Development 1985; 56: 853-860.

10 Rogan WJ, Gladen BC, McKinney JD, Carreras N, Hardy P, Thullen J, Tinglestad J, Tully M. Neonatal 
effects of transplacental exposure to PCBs and DDE. J Pediatr 1986; 109: 335-341.

11 Ballschmiter K, Bacher R, Mennel A, Fischer R, Riehle U, Swerev M. The determination of chlorinated biphenyls, chlorinated dibenzodioxins and chlorinated dibenzofurans by GC-MS. J High Resolut Chromatogr 1992; 15: 260-270.

12 Burse VW, Korver MP, Needham LL, Lapeza CR, Boozer EL, Head SL, et al. Gas chromatographic determination of polychlorinated biphenyls (as Aroclor 1254) in serum: Collaborative study. J Assoc Off Anal Chem 1989; 72: 649-659.

Van Rhijn JA, Traag WA, Van de Spreng PG, Tuinstra LGMTh. Simultaneous determination of planar chlorobiphenyls and polychlorinated dibenzo-p-dioxins and -furans in Dutch milk using isotope dilution and gas chromatography-high-resolution mass spectrometry. J Chromatogr 1993; 630: 297-306.

14 Tuinstra LGMTh, Van Rhijn JA et al. Method for the determination of dioxins, planar and other PCBs in human milk. In: Fiedler H, Frank $\mathrm{H}$ et al (eds). Organohalogen Compounds 11, Analytical Methods, Formation and Sources. Federal Environmental Agency, Austria 1993; pp 181-183.

NATO/CCMS. Pilot study on International Information Exchange on Dioxins and Related Compounds. International Toxicity Equivalency Factor (I-TEF). Method of Risk Assessment for Complex Mixtures of Dioxins and Related Compounds. Report number 176.

16 Ahlborg UG, Becking GC, Birnbaum LS, Brouwer A, Derks HJGM, Feeley M et al. Toxic Equivalency Factors for dioxin-like PCBs. Report on a WHO-ECEH and IPCS consultation, December 1993. Chemosphere 1994; 28 : 6.

17 World Health Organisation. Levels of PCBs, PCDDs, and PCDFs in breast milk: results of WHO-coordinated interlaboratory quality control studies and analytical field studies. FADL, Copenhagen. Environmental Health series 1989; 34.

18 Albers JMC, Kreis IA, Van Zoonen P, Liem AKD, Theelen RMC. Contamination of breastmilk with chlorinated organic compounds in the Netherlands, 1988. RIVM Rapport nr 638815002 (in Dutch, summary in English) 1993.

19 Huisman M, Koopman-Esseboom C, Touwen BCL, Van der Paauw CG, Sauer PJJ, Huisjes HJ, Muskiet FAJ, Boersma ER. Risk assessment of fetal exposure to polychlorinated biphenyls (PCBs). 13th Europian congress of perinatal medicine; Amsterdam (abstracts). J Perinat Med 1992;20 suppl 1:125.

20 Jensen AA. Transfer of chemical contaminants into human milk. In: Jensen AA, Slorach SA (eds). Chemical contaminants in human milk. CRC Press 1991: 9-19.

21 Van den Berg M, De Jongh J, Poiger H, Olson JR. The toxicokinetics and metabolism of polychlorinated dibenzo-p-dioxins (PCDDs) and dibenzofurans (PCDFs), and their relevance for toxicity. CRC Critical Reviews in Toxicology (in press).

22 Fürst $\mathrm{P}$ et al. PCDD and PCDF levels in human milk. Dependence on the period of lactation. Chemosphere 1989; 18: 439.

23 Norén K. Changes in the levels of organochlorine pesticides, polychlorinated biphenyls, dibenzo-p-dioxins and dibenzofurans in human milk from Stockholm, 1972-1985. Chemosphere 1988; 17:39-49. 